\title{
Kinematic Synthesis of Mechanism for System with a Technical Vision
}

\author{
Baurzhan Tultayev, Gani Balbayev, Algazy Zhauyt ${ }^{\mathrm{a}}$, Aidos Sultan and Aigerim Mussina \\ Gylym ordasy, Almaty 050013, Kazakhstan
}

\begin{abstract}
A solution to the problem of synthesizing an initial three-dimensional kinematic chain with spherical and rotary kinematic pairs is presented. It is shown that this chain can be used as a structural module for structural-kinematic synthesis of three-dimensional four-link motion generating lever mechanisms by the preset positions of the in-and output links. This paper affects the actual today's problem of optimal synthesis of spatial link mechanisms. In this regard, the task of developing methods for the synthesis of complex spatial link mechanisms with the desired laws of motion of the input and output elements allowing automatizing the implementation of all design phases with the use of computer is quite relevant. The authors develop machine-oriented method of structural and kinematic synthesis of spatial link mechanisms based on the use of spatial initial kinematic chains (IKC) realizing prescribed motions. A new approach to the design of spatial mechanisms is suggested, according to which the design process is based on the kinematic synthesis of four-link initial kinematic chain (IKC) and associable kinematic chains (AKC).
\end{abstract}

\section{Introduction}

By necessity of automation in the field of equipment at research optimum engineering of machine and mechanism according to criteria of force transfer are appeared new approaches of solution the task by Pencic et al. [1]. The new approach for drawing up algorithm and program at the optimum complex solution of the task according to criteria of force transfer of four and multilink mechanism is required for the solution of these tasks by Szkodny [2]. The way of the solution of the task at engineering of the initial kinematic chain (IKC) is presented and kinematic synthesis Jalón and Bayo [3] of its modifications of the four-link flat lever mechanism, and also regularity of the movement of multilink mechanism of the high class carrying out incoming and outgoing link on optimum criterion of force transfer. The research and engineering of regularity of the movement of the multilink mechanism carrying out incoming and outgoing link, and also the high-class mechanism is actual and demands comprehensive scientific and creative approach Lioa [4]. These papers demonstrate that four-link initial kinematic chains (IKC) may be used as a structural module with structural and kinematic synthesis of plain linkage mechanisms. Such an approach to the synthesis of plain mechanisms allows reducing the problem of their structural and kinematic synthesis to the solution of problem of IKC synthesis which is very useful for automation of mechanisms engineering. This paper testifies that specified approach may be applied to the problem of structural and kinematic synthesis of spatial

\footnotetext{
${ }^{a}$ E-mail: ali84jauit@mail.ru
} 
linkage mechanisms Schwerin [5]. The solution of the problem of synthesis of spatial IKC of SSS type (S spherical kinematic pairs) is represented and its use as a structural module with structural and kinematic synthesis of spatial linkage mechanisms as per predetermined positions of input and output links is shown. A method of solution the problem of IKC synthesis of SSS type is based on the introduction of two movable bodies invariably associated with the input and output links Nussibalieva et al. [6]. This papers contains new scientifically grounded results that solve the following problems: mathematical problems of synthesis of spatial four-link initial kinematic chain of general type with spherical pairs are formulated and solved on the basis of motion specification of two movable bodies relative to non-movable body; under different combinations of the given and required parameters of the spatial initial kinematic chain, its various modifications have been obtained Tultayev [7]. The reliability of the theoretical results is confirmed by numerical tests of all found theoretical laws. Numerical results of the kinematic synthesis indicate the reality and authenticity of regularities that were found theoretically, operating capability of the given algorithm of mechanisms synthesis and confirm the expected theoretical provisions Zhauyt et al. [8]. Raising the technical level of machines requires an expansion of technological capabilities of the equipment and devices of the existing structures, as well as their equipping with fundamentally new mechanisms. Creation and implementation of new structures of industrial robots, equipment for light industry, mining, and oil and gas industry requires the use of mechanisms with a complicated motion of actuating devices. In this regard, the task of developing methods for the synthesis of complex spatial link mechanisms with the desired laws of motion of the input and output elements allowing automatizing the implementation of all design phases with the use of computer is quite relevant Zhauyt et al. [9]. Presented article touches on the actual up to date problem of optimal synthesis of spatial link mechanisms. At the theoretical level, it is proposed a new approach to the design of spatial mechanisms, according to which the design process is based on the kinematic synthesis of four-link initial kinematic chain and associable kinematic chains or two-element chains. Article differs by serious problem statement, interesting theoretical study and practical test Zhauyt et al. [10]. The work contains new scientific results having theoretical and applied significance.

\section{Materials and Methods}

Problem statement: given $N$ of finite distant positions of two solids $Q_{1}$ and $Q_{2}$ :

$$
Q_{1}\left(X_{A}, Y_{A}, Z_{A}, \theta_{i}^{1}, \psi_{i}^{1}, \phi_{i}^{1}\right) Q_{2}\left(X_{D i}, Y_{D i}, Z_{D i}, \theta_{i}^{2}, \psi_{i}^{2}, \phi_{i}^{2}\right) i=\overrightarrow{1, N}
$$

where $\theta_{i}^{j}, \psi_{i}^{j}, \phi_{i}^{j}$ are fixed axis Eulerian angles $O X Y Z$ and $X_{D i}, Y_{D i}, Z_{D i}$ are coordinates of the point $D_{i}$ of the solid $Q_{2}$.

It is required to find such points in the fixed axis as $A\left(X_{A}, Y_{A}, Z_{A}\right), B\left(x_{B}, y_{B}, z_{B}\right)$ of the solid $Q_{1}$ and $C\left(x_{C}, y_{C}, z_{C}\right)$ of the solid $Q_{2}$, so that distance between the points $B$ and $C$ in all positions of the solids $Q_{1}$ and $Q_{2}$ is little different from some constant value $R$ (see Figure 1).

Problem solution: Let us introduce a weighted difference for the $i$ - th position of the solids in form:

$$
\Delta_{q_{i}}=\left|\overrightarrow{B_{i} C_{i}}\right|^{2}-R^{2}=\left(X_{C_{i}}-X_{B_{i}}\right)^{2}+\left(Y_{C_{i}}-Y_{B_{i}}\right)^{2}+\left(Z_{C_{i}}-Z_{B_{i}}\right)^{2}-R^{2} \quad i=\overrightarrow{1, N}
$$




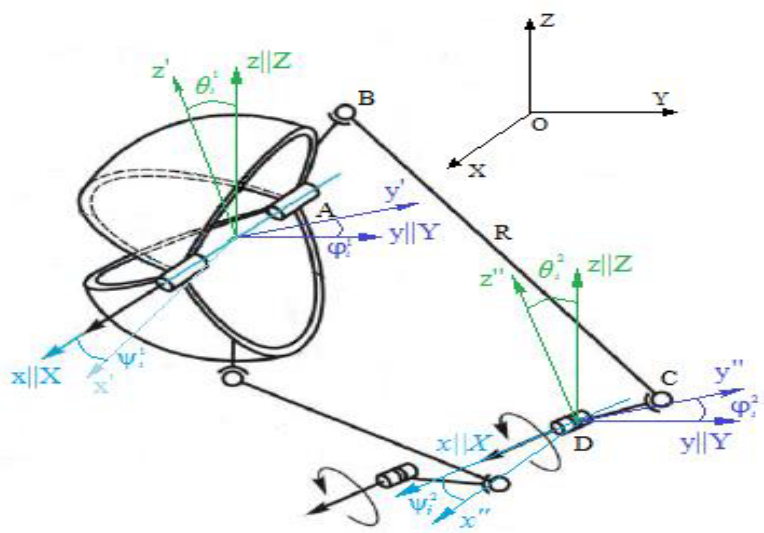

Figure 1. Equivalent RSSR four-link kinematic chain ABCD.

Relative to the fixed plane $Q$. Toughly connect with planes $Q, Q_{1}$ and $Q_{2}$ respectively, rectangular coordinate systems $O X Y Z, A_{x y z}$ and $D x y z$. Plane $Q_{1}$ rotates around the point $A$ with coordinates $X_{A}, Y_{A}, Z_{A}$, determined by the angle of rotation $\varphi$, and the plane $Q_{2}$ plane-parallel motion determined by the trajectory of its point $D$ with coordinates $X_{D}, Y_{D}, Z_{D}$ and angle of rotation $\psi$. To obtain a coordinated motion of two moving planes $Q_{1}$ and $Q_{2}$. It is necessary to impose geometric constraints on them, which can be done in two ways [1].

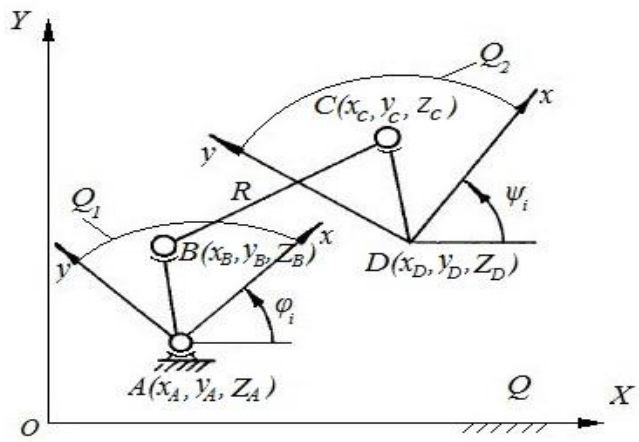

Figure 2. Equivalent SSS kinematic chain ABCD.

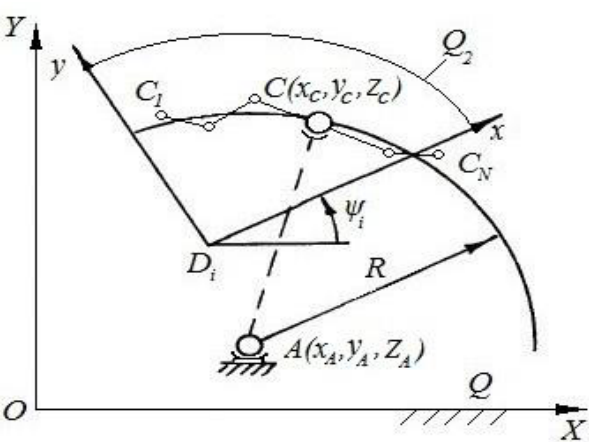

Figure 3. Equivalent $\mathrm{SS}$ kinematic chain $\mathrm{CD}$.

It is required to find on the plane $Q, Q_{1}$ and $Q_{2}$ respectively, such points $A\left(X_{A}, Y_{A}, Z_{A}\right)$, $B\left(x_{B}, y_{B}, z_{B}\right)$ and $C\left(x_{C}, y_{C}, z_{C}\right)$ that the distance between points $B$ or $C$. In all positions of the planes $Q_{1}$ and $Q_{2}$ The values determined by the relations differed little from a certain constant value $R$. Variable coordinates $X_{B i}, Y_{B i}, Z_{B i}$ points $B$ and $X_{C i}, Y_{C i}, Z_{C i}$ points $C$ in the $O X Y Z$ system are expressed in terms of their constant coordinates $x_{B}, y_{B}, z_{B}$ and $x_{C}, y_{C}, z_{C}$ in systems $A_{x y z}$ and $D x y z$ known linear transformation formulas (Figure 2) and (Figure 3): 


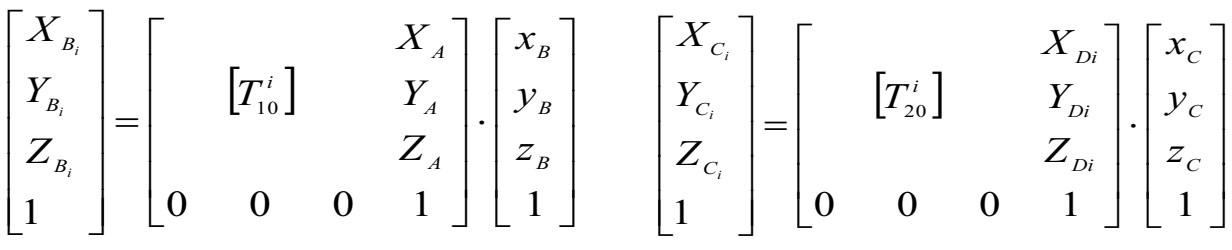

$$
\begin{aligned}
& T_{j o}^{i}=\left[\begin{array}{ccc}
e_{i 1}^{j} & e_{i 2}^{j} & e_{i 3}^{j} \\
m_{i 1}^{j} & m_{i 2}^{j} & m_{i 3}^{j} \\
n_{i 1}^{j} & n_{i 2}^{j} & n_{i 3}^{j}
\end{array}\right] \quad \begin{array}{l}
j=1,2 \\
i=1, N
\end{array}
\end{aligned}
$$

It is a function of ten parameters: $X_{A}, Y_{A}, Z_{A}, x_{B}, y_{B}, z_{B}, R, x_{C}, y_{C}, z_{C}$. By grouping these parameters in fours with the common parameter $R$, let us represent the weighted difference in three different forms [5-7]:

$$
\begin{aligned}
& \Delta_{q_{i}}^{(1)}=\left(\widetilde{X}_{A_{i}}-X_{A}\right)^{2}+\left(\widetilde{Y}_{A_{i}}-Y_{A}\right)^{2}+\left(\widetilde{Z}_{A_{i}}-Z_{A}\right)^{2}-R^{2} \\
& \Delta_{q_{i}}^{(2)}=\left(\widetilde{x}_{B_{i}}-x_{B}\right)^{2}+\left(\widetilde{y}_{B_{i}}-y_{B}\right)^{2}+\left(\widetilde{z}_{B_{i}}-Z_{B}\right)^{2}-R^{2} \\
& \Delta_{q_{i}}^{(3)}=\left(\widetilde{x}_{C_{i}}-x_{C}\right)^{2}+\left(\widetilde{y}_{C_{i}}-y_{C}\right)^{2}+\left(\widetilde{z}_{C_{i}}-Z_{C}\right)^{2}-R^{2}
\end{aligned}
$$

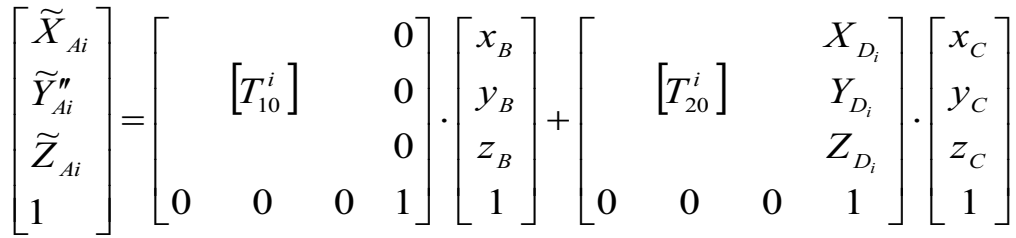

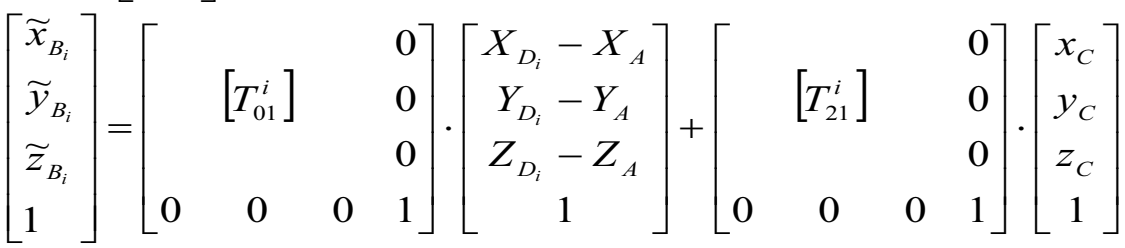

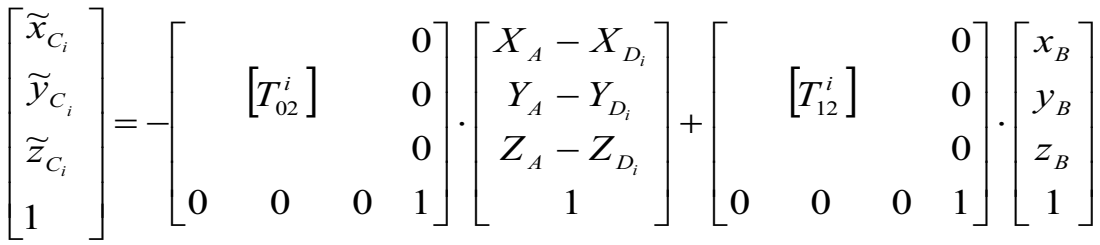

where $T_{k j}^{i}$ is the transfer matrix from the $k$ coordinate system to the $j$ system determined as:

$$
T_{01}^{i}=\left[T_{10}^{i}\right]^{T} \quad T_{02}^{i}=\left[T_{20}^{i}\right]^{T} \quad T_{21}^{i}=T_{01}^{i} \times T_{20}^{i} \quad T_{12}^{i}=T_{02}^{i} \times T_{10}^{i}
$$

the necessary conditions for minimum of the sum of squares of the weighted difference:

$$
S=\sum_{i=1}^{N}\left[\Delta_{q_{i}}^{(k)}\right]^{2} \quad(k=1,2, \ldots, 3)
$$

may be written as the following system of equations: 


$$
\begin{array}{llll}
\frac{\partial S}{\partial X_{A}}=0 & \frac{\partial S}{\partial Y_{A}}=0 & \frac{\partial S}{\partial Z_{A}}=0 & \frac{\partial S}{\partial R}=0 \\
\frac{\partial S}{\partial x_{B}}=0 & \frac{\partial S}{\partial y_{B}}=0 & \frac{\partial S}{\partial z_{B}}=0 & \frac{\partial S}{\partial R}=0 \\
\frac{\partial S}{\partial x_{C}}=0 & \frac{\partial S}{\partial y_{C}}=0 & \frac{\partial S}{\partial z_{C}}=0 & \frac{\partial S}{\partial R}=0
\end{array}
$$

From (11-13), considering (5-7) and (10), we obtain:

$$
\left.\begin{array}{c}
\sum_{i=1}^{N} \Delta_{q_{i}}^{(1)} \cdot\left(\widetilde{X}_{A_{i}}-X_{A}\right)=0 \\
\sum_{i=1}^{N} \Delta_{q_{i}}^{(1)} \cdot\left(\widetilde{Y}_{A_{i}}-Y_{A}\right)=0 \\
\sum_{i=1}^{N} \Delta_{q_{i}}^{(1)} \cdot\left(\widetilde{Z}_{A_{i}}-Z_{A}\right)=0 \\
\sum_{i=1}^{N} \Delta_{q_{i}}^{(1)} \cdot R=0
\end{array}\right\}
$$

Assume that $R \neq 0$. Then from the last equality of system (14), it follows that:

$$
\sum_{i=1}^{N} \Delta_{q_{i}}^{(1)}=0
$$

With provision for (15), the system of equations (14), takes the form:

$$
\sum_{i=1}^{N} \Delta_{q_{i}}^{(1)} \widetilde{X}_{A i}=0 \quad \sum_{i=1}^{N} \Delta_{q_{i}}^{(1)} \widetilde{Y}_{A i}=0 \quad \sum_{i=1}^{N} \Delta_{q_{i}}^{(1)} \widetilde{Z}_{A i}=0 \quad \sum_{i=1}^{N} \Delta_{q_{i}}^{(1)}=0
$$

By substituting expressions for $\Delta_{q_{i}}^{(1)}$ from (5-7) into system (16), we obtain:

$$
\left.\begin{array}{c}
\sum_{i=1}^{N}\left[\widetilde{X}_{A i}^{2} X_{A}+\widetilde{X}_{A i} \widetilde{Y}_{A i} Y_{A}+\widetilde{Z}_{A i} \widetilde{X}_{A i} Z_{A}+\frac{1}{2}\left(R^{2}-X_{A}^{2}-Y_{A}^{2}-Z_{A}^{2}\right) \widetilde{X}_{A i}\right]=\frac{1}{2} \sum_{i=1}^{N}\left(\widetilde{X}_{A i}^{2}+\widetilde{Y}_{A i}^{2}+\widetilde{Z}_{A i}^{2}\right) \widetilde{X}_{A i} \\
\sum_{i=1}^{N}\left[\widetilde{X}_{A i} \widetilde{Y}_{A i} X_{A}+\widetilde{Y}_{A i}^{2} Y_{A}+\widetilde{Z}_{A i} \widetilde{Y}_{A i} Z_{A}+\frac{1}{2}\left(R^{2}-X_{A}^{2}-Y_{A}^{2}-Z_{A}^{2}\right) \widetilde{Y}_{A i}\right]=\frac{1}{2} \sum_{i=1}^{N}\left(\widetilde{X}_{A i}^{2}+Y_{A i}^{2}+Z_{A i}^{2}\right) \widetilde{Y}_{A i} \\
\sum_{i=1}^{N}\left[\widetilde{Z}_{A i} \widetilde{X}_{A i} X_{A}+\widetilde{Y}_{A i} \widetilde{Z}_{A i} Y_{A}+\widetilde{Z}_{A i}^{2} Z_{A}+\frac{1}{2}\left(R^{2}-X_{A}^{2}-Y_{A}^{2}-Z_{A}^{2}\right) \widetilde{Z}_{A i}\right]=\frac{1}{2} \sum_{i=1}^{N}\left(\widetilde{X}_{A i}^{2}+\widetilde{Y}_{A i}^{2}+\widetilde{Z}_{A i}^{2}\right) \widetilde{Z}_{A i} \\
\sum_{i=1}^{N}\left[\widetilde{X}_{A i} X_{A}+\widetilde{Y}_{A i} Y_{A}+\widetilde{Z}_{A i} Z_{A}+\frac{1}{2}\left(R^{2}-X_{A}^{2}-Y_{A}^{2}-Z_{A}^{2}\right) \widetilde{X}_{A i}\right]=\frac{1}{2} \sum_{i=1}^{N}\left(\widetilde{X}_{A i}^{2}+\widetilde{Y}_{A i}^{2}+\widetilde{Z}_{A i}^{2}\right)
\end{array}\right\}
$$

System (17) is linear with respect to the variables $X_{A}, Y_{A}, Z_{A}$ and $H_{1}=\frac{1}{2}\left(R^{2}-X_{A}^{2}-Y_{A}^{2}-Z_{A}^{2}\right)$, thus it may be written as: 


$$
\left[\begin{array}{llll}
\sum_{i=1}^{N} \widetilde{X}_{A i}^{2} & \sum_{i=1}^{N} \widetilde{X}_{A i} \widetilde{Y}_{A i} & \sum_{i=1}^{N} \widetilde{X}_{A i} \widetilde{Z}_{A i} & \sum_{i=1}^{N} \widetilde{X}_{A i} \\
\sum_{i=1}^{N} \widetilde{X}_{A i} \widetilde{Y}_{A i} & \sum_{i=1}^{N} \widetilde{Y}_{A i} & \sum_{i=1}^{N} \widetilde{Y}_{A i} \widetilde{Z}_{A i} & \sum_{i=1}^{N} \widetilde{Y}_{A i} \\
\sum_{i=1}^{N} \widetilde{X}_{A i} \widetilde{Z}_{A i} & \sum_{i=1}^{N} \widetilde{Y}_{A i} \widetilde{Z}_{A i} & \sum_{i=1}^{N} \widetilde{Z}_{A i}^{2} & \sum_{i=1}^{N} \widetilde{Z}_{A i} \\
\sum_{i=1}^{N} \widetilde{X}_{A i} & \sum_{i=1}^{N} \widetilde{Y}_{A i} & \sum_{i=1}^{N} \widetilde{Z}_{A i} & N
\end{array}\right] \cdot\left[\begin{array}{c}
X_{A} \\
Y_{A} \\
Z_{A} \\
H_{1}
\end{array}\right]=\frac{1}{2}\left[\begin{array}{c}
\sum_{i=1}^{N} \widetilde{R}_{A_{i}}^{2} \widetilde{X}_{A_{i}} \\
\sum_{i=1}^{N} \widetilde{R}_{A_{i}}^{2} \widetilde{Y}_{A_{i}} \\
\sum_{i=1}^{N} \widetilde{R}_{A_{i}}^{2} \widetilde{Z}_{A_{i}} \\
\sum_{i=1}^{N} \widetilde{R}_{A_{i}}^{2}
\end{array}\right]
$$

where $\widetilde{R}_{A i}^{2}=\widetilde{X}_{A i}^{2}+\widetilde{Y}_{A i}^{2}+\widetilde{Z}_{A i}^{2}$. The solution to this system by Cramer's rule is as follows:

$$
\left(X_{A}, Y_{A}, Z_{A}, H_{1}\right)=\frac{1}{D_{1}}\left(D_{X_{A}}, D_{Y_{A}}, D_{Z_{A}}, D_{H_{1}}\right) \quad D_{1} \neq 0
$$

Similarly, from (11-13), considering (5-7) and (10), we obtain a system of linear equations in the unknowns $x_{B}, y_{B}, z_{B}, H_{2}$ :

$$
\left[\begin{array}{cccc}
\sum_{i=1}^{N} \widetilde{x}_{B i}^{2} & \sum_{i=1}^{N} \widetilde{x}_{B i} y_{B i} & \sum_{i=1}^{N} \widetilde{x}_{B i} \widetilde{z}_{B i} & \sum_{i=1}^{N} \widetilde{x}_{B i} \\
\sum_{i=1}^{N} \widetilde{x}_{B i} y_{B i} & \sum_{i=1}^{N} \widetilde{y}_{B i}^{2} & \sum_{i=1}^{N} \widetilde{y}_{B i} \widetilde{z}_{B i} & \sum_{i=1}^{N} \widetilde{y}_{B i} \\
\sum_{i=1}^{N} \widetilde{x}_{B i} \widetilde{z}_{B i} & \sum_{i=1}^{N} \widetilde{y}_{B i} \widetilde{z}_{B i} & \sum_{i=1}^{N} \widetilde{z}_{B i}^{2} & \sum_{i=1}^{N} \widetilde{z}_{B i} \\
\sum_{i=1}^{N} \widetilde{x}_{B i} & \sum_{i=1}^{N} \widetilde{y}_{B i} & \sum_{i=1}^{N} \widetilde{z}_{B i} & N
\end{array}\right] \cdot\left[\begin{array}{c}
x_{B} \\
y_{B} \\
z_{B} \\
H_{2}
\end{array}\right]=\frac{1}{2}\left[\begin{array}{l}
\sum_{i=1}^{N} \widetilde{R}_{B i}^{2} \widetilde{x}_{B i} \\
\sum_{i=1}^{N} \widetilde{R}_{B i}^{2} \widetilde{y}_{B i} \\
\sum_{i=1}^{N} \widetilde{R}_{B i}^{2} \widetilde{z}_{B i} \\
\sum_{i=1}^{N} \widetilde{R}_{B i}^{2}
\end{array}\right]
$$

By solving this system by Cramer's rule [5-7], we obtain:

$$
\left(x_{B}, y_{B}, z_{B}, H_{2}\right)=\frac{1}{D_{2}}\left(D_{x_{B}}, D_{y_{B}}, D_{z_{B}}, D_{H_{2}}\right) \quad D_{2} \neq 0
$$

From (11-13), considering (5-7) and (11-13), we obtain a system of linear equations in the unknowns $x_{C}, y_{C}, z_{C}, H_{3}$ :

$$
\left[\begin{array}{cccc}
\sum_{i=1}^{N} \widetilde{x}_{C i}^{2} & \sum_{i=1}^{N} \widetilde{x}_{C i} \widetilde{y}_{C i} & \sum_{i=1}^{N} \widetilde{x}_{C i} \widetilde{z}_{C i} & \sum_{i=1}^{N} \widetilde{x}_{C i} \\
\sum_{i=1}^{N} \widetilde{x}_{C i} \widetilde{y}_{C i} & \sum_{i=1}^{N} \widetilde{y}_{C i}^{2} & \sum_{i=1}^{N} \widetilde{y}_{C i} \widetilde{z}_{C i} & \sum_{i=1}^{N} \widetilde{y}_{C i} \\
\sum_{i=1}^{N} \widetilde{x}_{C i} \widetilde{z}_{C i} & \sum_{i=1}^{N} \widetilde{y}_{C i} \widetilde{z}_{C i} & \sum_{i=1}^{N} \widetilde{z}_{C i}^{2} & \sum_{i-1}^{N} \widetilde{z}_{C i} \\
\sum_{i=1}^{N} \widetilde{x}_{C i} & \sum_{i=1}^{N} \widetilde{y}_{C i} & \sum_{i=1}^{N} \widetilde{z}_{C i} & N
\end{array}\right] \cdot\left[\begin{array}{c}
x_{C} \\
y_{C} \\
z_{C} \\
H_{3}
\end{array}\right]=\frac{1}{2}\left[\begin{array}{c}
\sum_{i=1}^{N} \widetilde{R}_{C i}^{2} \widetilde{x}_{C i} \\
\sum_{i=1}^{N} \widetilde{R}_{C i}^{2} \widetilde{y}_{C i} \\
\sum_{i=1}^{N} \widetilde{R}_{C i}^{2} \widetilde{z}_{C i} \\
\sum_{i=1}^{N} \widetilde{R}_{C i}^{2}
\end{array}\right]
$$

From which we obtain $x_{C}, y_{C}, z_{C}, H_{3}$ :

$$
\left(x_{C}, y_{C}, z_{C}, H_{3}\right)=\frac{1}{D_{3}}\left(D_{x_{C}}, D_{y_{C}}, D_{z_{C}}, D_{H_{3}}\right) \quad D_{3} \neq 0
$$


Eliminating the first four unknowns $X_{A}, Y_{A}, Z_{A}, R$, based on formula (18), it is possible to bring system (11-13) to a system of six equations with six unknowns $x_{B}, y_{B}, z_{B}, x_{C}, y_{C}, z_{C}$, which is convenient to be given as:

$$
\begin{aligned}
\sum_{i=1}^{N} \Delta_{q_{i}}^{(1)} \frac{\partial \Delta_{q_{i}}^{(2)}}{\partial x_{B}}=0 & \sum_{i=1}^{N} \Delta_{q_{i}}^{(1)} \frac{\partial \Delta_{i}^{(3)}}{\partial x_{C}}=0 \\
\sum_{i=1}^{N} \Delta_{q_{i}}^{(1)} \frac{\partial \Delta_{q_{i}}^{(2)}}{\partial y_{B}}=0 & \sum_{i=1}^{N} \Delta_{q_{i}}^{(1)} \frac{\partial \Delta_{i}^{(3)}}{\partial y_{C}}=0 \\
\sum_{i=1}^{N} \Delta_{q_{i}}^{(1)} \frac{\partial \Delta_{q_{i}}^{(2)}}{\partial z_{B}}=0 & \sum_{i=1}^{N} \Delta_{q_{i}}^{(1)} \frac{\partial \Delta_{i}^{(3)}}{\partial z_{C}}=0
\end{aligned}
$$

Apparently, equations of this system are the same as the three equations of the thirteen degree in the three unknown functions given in the work by Zhauyt et al. [8-10], though in this case we have a system of six equations in six unknown functions. Solution of system (24) is labor-intensive task, so it is more effective to apply a search algorithm for the minimum of the function $S$ stated below.

Theorem: if $S_{1}^{(1)}, S_{2}^{(1)}, S_{3}^{(1)}$ minimum values of sum $S$, determined for N position corresponding to the pairs of points:

$$
\begin{aligned}
& \left\{B^{(0)}\left(x_{B}^{\prime(0)}, y_{B}^{\prime(0)}, z_{B}^{\prime(0)}\right) \in Q_{1}, C^{(0)}\left(x_{C}^{\prime \prime(0)}, y_{C}^{\prime \prime(0)}, z_{C}^{\prime \prime(0)}\right) \in Q_{2}\right\} \\
& \left\{A^{(1)}\left(x_{A}^{(1)}, y_{A}^{(1)}, z_{A}^{(1)}\right) \in Q, C^{(0)}\left(x_{C}^{\prime \prime(0)}, y_{C}^{\prime(0)}, z_{C}^{\prime \prime(0)}\right) \in Q_{2}\right\} \\
& \left\{A^{(1)}\left(x_{A}^{(1)}, y_{A}^{(1)}, z_{A}^{(1)}\right) \in Q, B^{(1)}\left(x_{B}^{\prime(1)}, y_{B}^{\prime(1)}, z_{B}^{\prime(1)}\right) \in Q_{1}\right\}
\end{aligned}
$$

such, that

$$
\begin{aligned}
& S_{1}^{(1)}=\min _{X_{A}, Y_{A}, Z_{A}, R} S\left[X_{A}, Y_{A}, Z_{A}, R, x_{B}^{(0)}, y_{B}^{(0)}, z_{B}^{\prime(0)}, x_{C}^{\prime \prime(0)}, y_{C}^{\prime \prime(0)}, z_{C}^{\prime \prime(0)}\right]= \\
& =S\left[X_{A}^{(1)}, Y_{A}^{(1)}, Z_{A}^{(1)}, R_{1}^{(1)}, x_{B}^{\prime(0)}, y_{B}^{(0)}, z_{B}^{\prime(0)}, x_{C}^{\prime \prime(0)}, y_{C}^{\prime \prime(0)}, z_{C}^{\prime \prime(0)}\right] \\
& S_{2}^{(1)}=\min _{x_{B}^{\prime}, y_{B}^{\prime}, z_{B}^{\prime}, R} S\left[X_{A}^{(1)}, Y_{A}^{(1)}, Z_{A}^{(1)}, R, x_{B}^{\prime}, y_{B}^{\prime}, z_{B}^{\prime}, x_{C}^{\prime(0)}, y_{C}^{\prime \prime(0)}, z_{C}^{\prime \prime(0)}\right]= \\
& =S\left[X_{A}^{(1)}, Y_{A}^{(1)}, Z_{A}^{(1)}, R_{2}^{(1)}, x_{B}^{(1)}, y_{B}^{(1)}, z_{B}^{(1)}, x_{C}^{\prime(0)}, y_{C}^{\prime(0)}, z_{C}^{\prime(0)}\right] \\
& S_{3}^{(1)}=\min _{x_{C}^{\prime \prime}, y_{C}^{\prime \prime}, z_{C}^{\prime \prime}, R} S\left[X_{A}^{(1)}, Y_{A}^{(1)}, Z_{A}^{(1)}, R, x_{B}^{\prime(1)}, y_{B}^{(1)}, z_{B}^{\prime(1)}, x_{C}^{\prime \prime}, y_{C}^{\prime \prime}, z_{C}^{\prime \prime}\right]
\end{aligned}
$$

then $S_{3}^{(1)} \leq S_{2}^{(1)} \leq S_{1}^{(1)}$.

Proof: The system 20 when $D_{1} \neq 0$ defines for any points $B^{(0)} \in Q_{1}$ and $C^{(0)} \in Q_{2}$ only one solution $\left(X_{A}^{(1)}, Y_{A}^{(1)}, Z_{A}^{(1)}, R_{1}^{(1)}\right)$ corresponding to minimum sum $S$ of variables $X_{A}, Y_{A}, Z_{A}$ and $R$. Therefore, the following equality can be written for a given pair $\left(B^{(0)}, C^{(0)}\right)$

$$
\begin{aligned}
& S_{1}^{(1)}=\min _{X_{A}, Y_{A}, Z_{A}, R} S\left[X_{A}, Y_{A}, Z_{A}, R, x_{B}^{\prime(0)}, y_{B}^{\prime(0)}, z_{B}^{\prime(0)}, x_{C}^{\prime \prime(0)}, y_{C}^{\prime \prime(0)}, z_{C}^{\prime \prime(0)}\right]= \\
& =S\left[X_{A}^{(1)}, Y_{A}^{(1)}, Z_{A}^{(1)}, R_{1}^{(1)}, x_{B}^{\prime(0)}, y_{B}^{\prime(0)}, z_{B}^{\prime(0)}, x_{C}^{\prime \prime(0)}, y_{C}^{\prime \prime(0)}, z_{C}^{\prime \prime(0)}\right]
\end{aligned}
$$


Similarly, the system 22 when $D_{2} \neq 0$ defines for the points $A^{(1)} \in Q$ and $C^{(0)} \in Q_{2}$ only one solution $\left(x_{B}^{\prime(1)}, y_{B}^{\prime(1)}, z_{B}^{\prime(1)}, R_{2}^{(1)}\right)$ corresponding to minimum sum $S$ of variables $x_{B}^{\prime}, y_{B}^{\prime}, z_{B}^{\prime}$ and $R$ :

$$
\begin{aligned}
& S_{2}^{(1)}=S\left[X_{A}^{(1)}, Y_{A}^{(1)}, Z_{A}^{(1)}, R_{2}^{(1)}, x_{B}^{(1)}, y_{B}^{\prime(1)}, z_{B}^{(1)}, x_{C}^{\prime \prime(0)}, y_{C}^{\prime \prime(0)}, z_{C}^{\prime \prime(0)}\right]= \\
& =\min _{x_{B}^{\prime}, y_{B}^{\prime}, z_{B}^{\prime}, R} S\left[X_{A}^{(1)}, Y_{A}^{(1)}, Z_{A}^{(1)}, R, x_{B}^{\prime}, y_{B}^{\prime}, z_{B}^{\prime}, x_{C}^{\prime \prime(0)}, y_{C}^{\prime \prime(0)}, z_{C}^{\prime \prime(0)}\right] \leq \\
& \leq \min _{x_{B}^{\prime}, y_{B}^{\prime}, z_{B}^{\prime}, R} S_{1}^{(1)}\left[X_{A}^{(1)}, Y_{A}^{(1)}, Z_{A}^{(1)}, R_{1}^{(1)}, x_{B}^{\prime}, y_{B}^{\prime}, z_{B}^{\prime}, x_{C}^{\prime(0)}, y_{C}^{\prime \prime(0)}, z_{C}^{\prime \prime(0)}\right] \leq S_{1}^{(1)}
\end{aligned}
$$

The system 24 when $D_{3} \neq 0$ defines for the points $A^{(1)} \in Q$ and $B^{(1)} \in Q_{1}$ only one solution $\left(x_{C}^{\prime \prime(1)}, y_{C}^{\prime \prime(1)}, z_{C}^{\prime \prime(1)}, R_{3}^{(1)}\right)$ corresponding to minimum sum $S$ of variables $x_{C}^{\prime \prime}, y_{C}^{\prime \prime}, z_{C}^{\prime \prime}$ and $R$ :

$$
\begin{aligned}
& S_{3}^{(1)}=S\left[X_{A}^{(1)}, Y_{A}^{(1)}, Z_{A}^{(1)}, R_{3}^{(1)}, x_{B}^{(1)}, y_{B}^{(1)}, z_{B}^{(1)}, x_{C}^{\prime(1)}, y_{C}^{\prime \prime(1)}, z_{C}^{\prime(1)}\right]= \\
& =\min _{x_{C}^{\prime \prime}, y_{C}^{\prime \prime}, z_{C}^{\prime \prime}, R} S\left[X_{A}^{(1)}, Y_{A}^{(1)}, Z_{A}^{(1)}, R, x_{B}^{(1)}, y_{B}^{(1)}, z_{B}^{(1)}, x_{C}^{\prime \prime}, y_{C}^{\prime \prime}, z_{C}^{\prime \prime}\right] \leq \\
& \leq \min _{x_{C}^{\prime \prime}, y_{C}^{\prime \prime}, z_{C}^{\prime \prime}, R} S_{2}^{(1)}\left[X_{A}^{(1)}, Y_{A}^{(1)}, Z_{A}^{(1)}, R_{2}^{(1)}, x_{B}^{(1)}, y_{B}^{(1)}, z_{B}^{(1)}, x_{C}^{\prime \prime}, y_{C}^{\prime \prime}, z_{C}^{\prime \prime}\right] \leq S_{2}^{(2)} .
\end{aligned}
$$

Consequently, $S_{3}^{(1)} \leq S_{2}^{(1)} \leq S_{1}^{(1)}$.

Algorithm for minimizing the function $S$ :

i. Give arbitrarily initial points $B^{(0)} \in Q_{1}, C^{(0)} \in Q_{2}$ and check the condition $D_{1} \neq 0$.

ii. Solve the system of linear equations (19) and determine $X_{A}^{(1)}, Y_{A}^{(1)}, Z_{A}^{(1)}, R_{1}^{(1)}$.

iii. Give points $A^{(1)} \in Q, C^{(0)} \in Q_{2}$ and check the condition $D_{2} \neq 0$.

iv. Solve the system of equations (21) and determine $x_{B}^{(1)}, y_{B}^{(1)}, z_{B}^{(1)}, R_{2}^{(1)}$.

v. Give points $A^{(1)} \in Q, B^{(1)} \in Q_{1}$ and check the condition $D_{3} \neq 0$.

vi. Solve the system of equations (23) and determine $x_{C}^{\prime(1)}, y_{C}^{\prime(1)}, z_{C}^{\prime(1)}, R_{3}^{(1)}$.

vii. Cyclically repeat steps i-vi, taking into account $A^{(i)}=A^{(i+1)}, B^{(i)}=B^{(i+1)}, C^{(i)}=C^{(i+1)}$, $i=0,1,2 \ldots$

By applying the algorithm, we obtain a decreasing sequence of values of the objective function $S_{1}^{(1)}, S_{2}^{(1)}, S_{3}^{(1)}, S_{1}^{(2)}, S_{2}^{(2)}, S_{3}^{(2)}, S_{1}^{(3)}, S_{2}^{(3)}, S_{3}^{(3)}$ which has a limit equal to the value of the function $S$ at the point of local minimum. When satisfying the inequality

$$
\max \left(\left|R^{(i)}-R^{(i-1)}\right|,\left|X_{A}^{(i)}-X_{A}^{(i-1)}\right|,\left|Y_{A}^{(i)}-Y_{A}^{(i-1)}\right|, \quad\left|Z_{A}^{(i)}-Z_{A}^{(i-1)}\right|\right) \leq \varepsilon
$$

\section{Results and discussion}

Judging from deviations, the points $C_{1}, C_{2}, C_{3}$ are the exact spherical points (found with the given measure of inaccuracy), and $C_{4}$ and $C_{5}$ are approximate points. To solve this problem, a Visual Basic program has been written. The results of the problem solution are given in Table 1. The calculations have been made within the range: 
$-1.2<\mathrm{MJ}<0.6 ;-1.2<\mathrm{NJ}<0.6 ;-1.2<\mathrm{KJ}<0.6$. With a pitch $d m_{j}=0.2$. In the entire range of calculations, the process of calculation is concurrent. The global minimum is equal to $S_{\min }=0.0042921$ and it is achieved at: $\mathrm{LL}=3, \mathrm{KK}=2, \mathrm{II}=2$. In Table 1, only a part of results in the neighborhood of the global minimum is shown. Its value is highlighted in bold frame in Table 1 . Below, the results of calculation of the objective function in form of carpet plots and 3D plots in Figure 4, Figure 5, Figure 6, and Figures 7 are given.

Table 1. Assigned positions of the body for synthesis of a single movable mechanism with $N=7$.

\begin{tabular}{|c|c|c|c|c|c|c|}
\hline \multirow{2}{*}{$\begin{array}{c}\text { Position } \\
\text { № }(i)\end{array}$} & \multirow{2}{*}{$x_{O i}$} & $y_{O i}$ & \multirow{2}{*}{$z_{O i}$} & \multicolumn{3}{|c|}{ Euler angles [deg] } \\
\cline { 5 - 7 } & & & & $\theta_{i}^{j}$ & $\psi_{i}^{j}$ & $\phi_{i}^{j}$ \\
\hline 1 & 0.30 & 0.12 & 0.01 & 0 & 0 & 0 \\
\hline 2 & 0.35 & 0.17 & 0.24 & 28 & 35 & 17 \\
\hline 3 & 0.44 & 0.21 & 0.25 & 34 & 38 & 5 \\
\hline 4 & 0.51 & 0.15 & 0.32 & 17 & 24 & 12 \\
\hline 5 & 0.50 & 0.30 & 0.45 & 50 & 50 & 21 \\
\hline 6 & 0.60 & 0.25 & 0.41 & 45 & 33 & 24 \\
\hline 7 & 0.55 & 0.32 & 0.35 & 0 & 0 & 0 \\
\hline
\end{tabular}

Graphics $S$

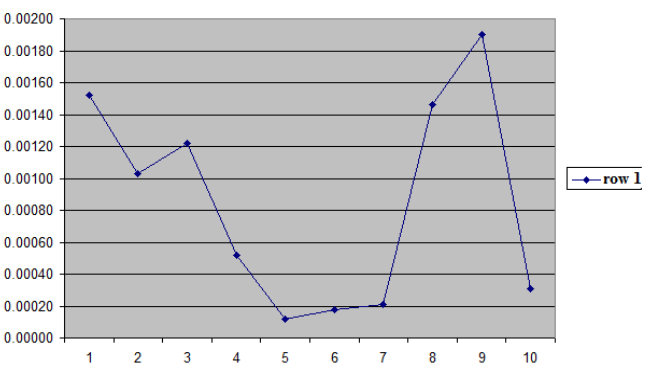

Figure 4. Computed plot of points $S$.

Graphics of function $\mathrm{S}=\mathrm{S}(\mathrm{R})$

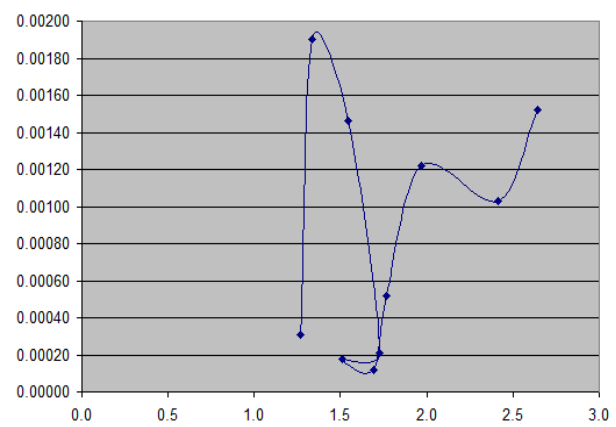

Figure 6. Computed plot of function points $S=S(R)$.

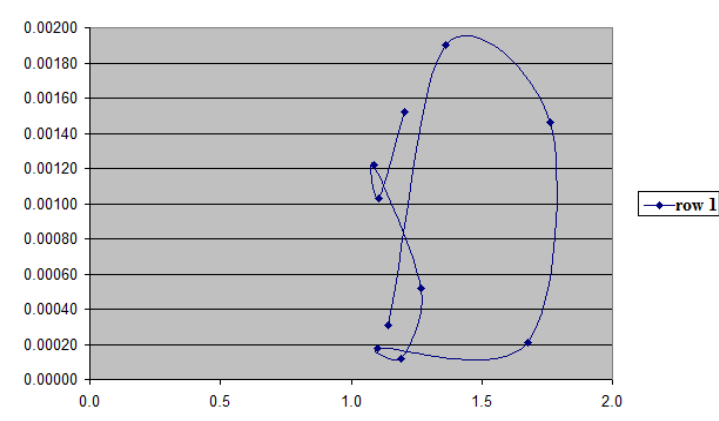

Figure 5. Computed plot of function points $S=S\left(Y_{A}\right)$.

3D graphics of function $S=S\left(x_{B}, y_{B}, Z B\right)$

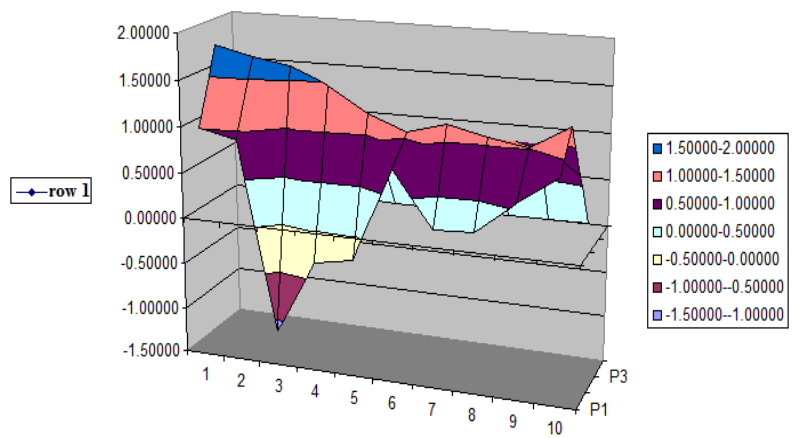

Figure 7.3D graphics of function $S=S\left(x_{B}, y_{B}, z_{B}\right)$.

According to algorithm the value of the global minimum $\mathrm{S}_{\min }=0.004292$ has been determined as well as the value of coordinates of the global minimum: 


$$
\begin{array}{ccc}
x_{B}=-0.227631, & x_{C}=0.652906, & X_{A}=Z_{A}=0, \\
y_{B}=1.21314, & y_{C}=0.639147, & Y_{A}=1.189776, \\
z_{B}=0.609801, & z_{C}=0.469694, & R==1.69127 .
\end{array}
$$

\section{Conclusions}

As can be concluded, the use of one and the same objective function being generated for synthesis of IKC and its modification allows automating of the process of synthesis of spatial linkage mechanisms through predetermined positions of the input and output links of the mechanism. In summary, in the synthesis of IKC with spherical kinematic pairs through predetermined positions of the input and output links of a mechanism when two adjacent links of IKC tend to infinity, it is necessary to replace the spherical kinematic pair for a plain or cylindrical one. In such a case, the synthesized mechanism takes form of a spatial link mechanism after determining the required parameters.

\section{References}

1. Pencic M., Cavic M., Rackov M., Borovac B. and Lu Z. Drive system of the robot eyeballs and eyelids with 8 DOFs. Mechanisms and Machine Science, 57, pp. 47-56 (2018)

2. T. Szkodny Dynamics of industrial robot manipulators. Mechanism and Machine Theory, 30, pp. 1057-1072 (1995)

3. J.G. de Jalón, E. Bayo Kinematic and Dynamic Simulation of Multibody Sytems: The Real-Time Challenge. Springer-Verlag, NewYork, (1994)

4. M.D. Lioa, V. Cossalter, R. Lot On the use of natural coordinates in optimal synthesis of mechanisms. Mechanism and Machine Theory, 35, pp. 1367-1389 (2000)

5. R.V. Schwerin Multibody System Simulation Numerical Methods, Algorithms, and Software. Springer-Verlag, Berlin Heidelberg, (1999)

6. Nussibalieva A., Mussina A., Carbone G., Tultayev B., Balbayev G. Neurorobotic Investigation by using a basic vestibulo-ocular reflex system algorithm. New Trends in Medical and Service Robotics: Advances in Theory and Practice, pp. 126-134 (2018)

7. Baurzhan Tultayev, Gani Balbayev, Algazy Zhauyt A kinematic analysis of flat leverage mechanism of the fourth class for manipulators. Materials Science and Engineering, 230, 2017, pp. 1-7 (2017)

8. Algazy Zhauyt, Kuanyshkali Alipov, Aizhan Sakenova, Adilet Zhankeldi, Raushan Abdirova, Zhastalap Abilkaiyr The synthesis of four-bar mechanism. Vibroengineering PROCEDIA, 10, pp. 486-491 (2016)

9. Algazy Zhauyt, Gulnar Mamatova, Kuanysh Alipov, Aizhan Sakenova, Raushan Abdirova The kinematic analysis of flat lever mechanisms with application of vector calculation. Vibroengineering PROCEDIA, 8, pp. 1-5 (2016)

10. Algazy Zhauyt, Indira Zhahanova, Gulbarshyn Smailova, Ulbala Murzakhmetova, Lazzat Kurmangalieva, Akmaral Kurenbay The kinematic analysis of the third class mechanism. Vibroengineering PROCEDIA, 12, pp. 208-212 (2017) 